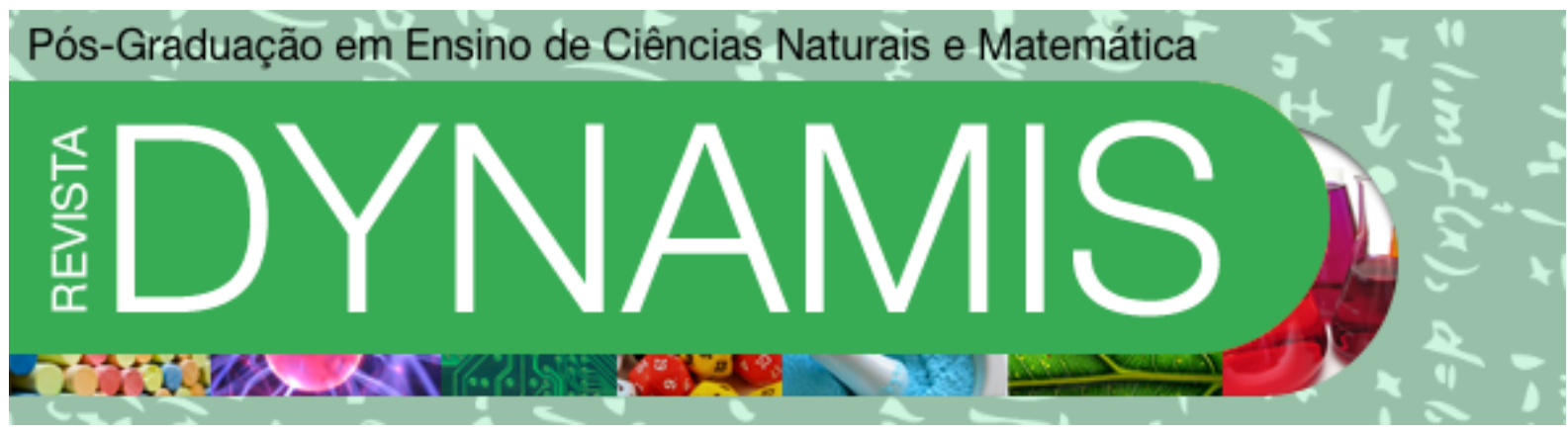

\title{
CARACTERIZAÇÃO DE MODELOS PEDAGÓGICOS DE EDUCAÇÃO SUPERIOR A DISTÂNCIA: UM ESTUDO DE CASO COLETIVO
}

Characterization of Pedagogical Models of Distance Education: a Collective Case Study

Selma dos Santos Rosa

Programa de pós-graduação em Educação Científica e Tecnológica

Universidade Federal de Santa Catarina - UFSC

selmadossantosrosa@gmail.com

José André Peres Angotti José André Peres Angotti

Programa de pós-graduação em Educação Científica e Tecnológica

Universidade Federal de Santa Catarina - UFSC

zeangotti@gmail.com 


\section{Resumo}

Este artigo constitui-se na descrição das trilhas metodológicas de uma investigação de doutorado em andamento. Reflete-se sobre como uma pesquisa de caráter qualitativo, baseada na análise de conteúdo, na revisão bibliográfica e no estudo de caso coletivo, contribui para caracterizar Modelos Pedagógicos de Educação a Distância (EaD) de nível superior. Para tal, se considerou promissor desenvolve-la à luz do significado da experiência educacional vivida por docentes, em suas próprias realidades. Na base ontológica, predomina a construção do conhecimento a partir da experiência desses sujeitos, cujo resultado constituiu a essência dos dados empíricos. Selecionaram-se 3 unidades de análise e 2 cursos de áreas diferentes com a proposição de caracterizar Modelos de EaD brasileiros, e também uma unidade de análise portuguesa com a proposição de buscar contribuições externas. Nas considerações finais enfatiza-se as limitações e as possibilidades que emergiram ao longo da pesquisa, no que tange a metodologia utilizada.

Palavras-chave: Modelos de Educação a Distância, Estudo de caso coletivo, Metodologia de pesquisa.

\section{Abstract}

This article constitutes in the description of trials methodological of a doctoral research in progress. It is reflected as a qualitative research based on content analysis, in the literature review and in the collective case study contributes to characterize Pedagogical Models of Distance Education higher level. For this purpose, it was considered a promising way develop it in the light of the meaning of educational experience experienced by teachers in their own realities. In ontological basis, predominate the construction of knowledge from the experience of these individuals, whose result is the essence of the empirical data. We selected three units of analysis and two courses from different areas with the proposition characterize Models Brazilians of Distance Education, and a unit of Portugal analysis with the proposition of seeking external contributions. In the concluding are emphasized the limitations and possibilities that emerged during the research, regarding the methodology used.

Keywods: Models of Distance Education, Collective case study, Research methodology. 


\section{Introdução}

No Brasil, vislumbra-se que uns dos desafios postos aos Modelos Pedagógicos de Educação a Distância (EaD) no ensino superior é transcender do discurso de Modelos com potencialidades voltadas à interação e à colaboração pedagógica para a prática de operacionalizá-lo na realidade das Universidades públicas. Essa configuração, em que a interação e a colaboração virtual imperam, caracteriza a EaD online, a qual tem sido indicada com potenciais para alterar processos e métodos educacionais. Na pesquisa ora apresentada, parte-se do princípio de que a EaD abarca a Educação online e também a tradicional, sendo que a primeira tem se destacado, ultimamente, devido à adesão cada vez maior às Tecnologias de Informação e Comunicação (TIC) a favor da ascensão da segunda, com menos predomínio ao uso desses recursos, e que essa característica conduzirá a EaD online a ocupar um espaço central na Educação nos próximos anos.

$\mathrm{Na}$ direção apontada, experiências nacionais e internacionais relatam o potencial da $\mathrm{EaD}$ online, bem como a intencionalidade e as dificuldades das Instituições de Ensino Superior (IES) de se distanciarem dos modelos da primeira e da segunda geração da EaD. Esses modelos, discutidos amplamente por Moore e Kearsley (2007), Garrison (2000) Garrison \& Anderson (2003), Gomes (2008), entre outros, se aproximam das gerações em que as TIC imperam, ou seja, da terceira, quarta, quinta e sexta gerações (GOMES, 2008). Além disso, tem-se constatado que as contribuições teóricas sobre a modalidade de ensino a distância são fundamentais, mas que concretizá-las no anônimo do dia a dia da prática de quem a consolida é uma ação com alto grau de dificuldade, que exige estratégias específicas para os diversos campos de conhecimento. Assim, o desafio emergente que as instituições enfrentam é adaptar seus projetos de curso a distância às características peculiares a essa modalidade de ensino. Como consequência, aspectos epistemológicos, organizacionais, tecnológicos e pedagógicos já estabelecidos requerem atenção e, se necessário, serem submetidos a readaptações na perspectiva de manter uma oferta de cursos com qualidade, uma vez que o resultado incidirá na formação das pessoas envolvidas e interferirá no sistema educacional, tanto no presencial quanto no a distância.

No sentido exposto, a presente pesquisa soma-se às que buscam contribuir com Modelos de EaD. Este artigo, por sua vez, constitui-se na descrição das Trilhas Metodológicas de uma investigação de doutorado em andamento sobre Modelos de Educação a Distância $(\mathrm{EaD})$ de nível superior, sob perspectivas e experiências de docentes, vinculada à linha Mídias e Ensino de Ciências do Programa de Pós-Graduação em Educação Científica e Tecnológica, da Universidade Federal de Santa Catarina (PPGECT/UFSC).

$\mathrm{Na}$ investigação mencionada, tem-se a proposição de analisar como as TIC digitais (re)configuram Modelos Pedagógicos de $\mathrm{EaD}$ tradicionais para online no ensino superior. No decorrer da pesquisa, apresentam-se características de Modelos de EaD brasileiros e do Modelo de Educação online da Universidade Aberta (UAb) de Portugal que conduzem à compreensão dessa (re)configuração. Considera-se que a EaD tradicional (re)configura-se para online na medida em que:

- a maioria do tempo de ensino e de aprendizagem se realize online;

- os recursos didáticos digitais (online) substituam os materiais impressos;

- a interação e a colaboração virtual pedagógica prevaleçam sobre a presencial, seguramente em termos de quantidade e potencialmente em termos de qualidade; e

- haja a proeminência da combinação da comunicação virtual síncrona e assíncrona, associando a aprendizagem individual e a colaborativa. 
Pelo exposto, tem-se, neste artigo, a proposição de descrever a Trilha Metodológica da investigação supracitada. Para tanto, questiona-se: como uma pesquisa de caráter qualitativo, baseada na análise de conteúdo, na revisão bibliográfica e no estudo de caso coletivo, contribui para caracterizar Modelos Pedagógicos de EaD de nível superior?

$\mathrm{Na}$ próxima seção, descrevem-se as Trilhas Metodológicas da pesquisa e, ao final, apresentam-se considerações finais, incluindo as limitações e possibilidades que emergiram ao longo da pesquisa, no que tange a metodologia de pesquisa utilizada.

\section{Trilhas Metodológicas}

Esta pesquisa, de natureza qualitativa, cuja fundamentação encontra-se na análise do conteúdo e dos seus significados, fundamenta-se no estudo de um problema com a finalidade de interpretar fenômenos a partir da exploração de informações e diálogos aferidos por sujeitos que deles participam ou estejam envolvidos, de alguma maneira (DENZIN \& LINCOLN, 2006; COUTINHO, 2011). Para tal se considera um caminho promissor desenvolver esta pesquisa à luz do significado da experiência (educacional) vivida pelos docentes de cursos a distância, em suas próprias realidades. Assim, na base ontológica desta pesquisa, predomina a construção do conhecimento a partir da experiência desses sujeitos, cujo resultado constituirá a essência dos dados empíricos, conforme se expõe a seguir.

A pesquisa relatada constitui-se de uma revisão bibliográfica e de um estudo de caso coletivo, realizados, na maioria do tempo, concomitantemente. A primeira conjugou levantamento, organização e classificação de pesquisas afins disponíveis em livros, artigos, dissertações e teses, com ênfase nos Modelos de EaD, Educação online, aprendizagem colaborativa, interação virtual e tecnologias educacionais. Dados dos projetos dos cursos das IES brasileiras, do Sistema Universidade Aberta do Brasil (UAB), do guia do Modelo de EaD da UAb, de Portugal, e de documentos legislativos brasileiros também se constituem fontes empíricas para esta investigação. A revisão bibliográfica conduziu ao planejamento, à revisão e à confirmação: do problema de pesquisa, da definição das técnicas de coletas de dados, da composição das questões do guia de entrevista e dos sujeitos e das instituições envolvidas.

Para a realização do estudo de caso coletivo de caráter qualitativo, seguiram-se as orientações de Stake (2005), segundo o qual, nesse tipo de abordagem, predomina o estudo de vários casos conjuntamente para obter melhor contextualização e compreensão de um fenômeno, uma população ou uma condição geral. Na pesquisa relatada, por exemplo, selecionaram-se 3 unidades de análise (casos) e 2 cursos de áreas diferentes com a proposição de caracterizar Modelos de $\mathrm{EaD}$ brasileiros, e também uma unidade de análise (caso) portuguesa com a proposição de buscar contribuições externas, ou seja, a ênfase não se desloca para as IES ou um curso de licenciatura específico, mas para o que eles revelaram sobre Modelos de EaD.

Estudo de casos coletivos (STAKE, 2005) ou múltiplos (YIN, 2005) é considerado mais consistente do que estudo de caso único. Ao utilizá-lo, o pesquisador não busca casos representativos de uma população, para a qual deseja generalizar os resultados, mas, a partir de um conjunto particular de resultados, pode encontrar generalizações teóricas aplicáveis em outros contextos. No entanto, Stake (2005) alerta para o fato de que há a possibilidade de as generalizações desviarem a atenção do pesquisador sobre as particularidades de cada caso, as quais podem prover contribuições relevantes, entretanto, não generalizáveis.

Segundo Yin (2005), o estudo de caso deve ser utilizado em pesquisas que se constituem de dados empíricos que buscam examinar acontecimentos contemporâneos e definidas com problemas de pesquisa que buscam investigar o "como" ou o "porquê" desses acontecimentos. Da mesma forma, permite incluir, em seus métodos de coleta de dados, 38 
diversos tipos de recursos, entre eles, a observação direta do fenômeno ou entrevistas com pessoas com ele envolvidas. É propício, ainda segundo o mesmo autor, quando se quer investigar condições contextuais, na perspectiva de que podem ser altamente pertinentes ao fenômeno em estudo. Nesse sentido, na pesquisa relatada, questiona-se: como as Tecnologias de Informações e Comunicação (TIC) digitais (re)configuram Modelos Pedagógicos de EaD tradicionais para online no ensino superior?

Merriam (1988, apud ANDRÉ, 2005), após revisar diversas definições de estudo de caso, o entende como particularidade em estudar um foco, um programa ou um fenômeno e a ele confere quatro características marcantes. Segundo André (2005, p. 18), essas características são: as revelações sobre o fenômeno ou sobre o que ele representa conferem importância ao caso em si e, por isso, constitui-se " um tipo de estudo adequado para investigar problemas práticos, questões que emergem do cotidiano"; o produto final de um estudo de caso consiste em uma descrição densa da situação investigada; caracteriza-se como heurístico, por possibilitar a revelação de novos significados e estender ou confirmar a compreensão do leitor sobre algo já conhecido; e comumente estudos de casos baseiam-se na lógica indutiva que, segundo Merriam, (1988, p.13, apud ANDRÉ, 2005, p. 18), volta-se para a "descoberta de novas relações, conceitos, compreensão, mais do que verificação ou hipótese pré-definida".

No quadro a seguir, apresenta-se uma síntese da constituição da metodologia desta pesquisa:

Quadro 1 - constituição da metodologia da pesquisa

\begin{tabular}{|l|l|l|}
\hline \multicolumn{2}{|l|}{ Quanto } & Autores \\
\hline $\begin{array}{l}\text { Ao procedimento e } \\
\text { métodos }\end{array}$ & $\begin{array}{l}\text { Estudo de caso coletivo e revisão } \\
\text { bibliográfica }\end{array}$ & $\begin{array}{l}\text { Stake (2005); André (2005); Yin } \\
\text { (2005); Gil (2008); Coutinho } \\
(2011)\end{array}$ \\
\hline $\begin{array}{l}\text { À natureza do } \\
\text { problema, metas e } \\
\text { marco teórico }\end{array}$ & Qualitativa, Teórico-exploratória & $\begin{array}{l}\text { Denzin \& Lincoln (2006); Gil } \\
\text { (2008); Coutinho (2011) }\end{array}$ \\
\cline { 1 - 2 } $\begin{array}{l}\text { Aos instrumentos de } \\
\text { coleta de dados }\end{array}$ & $\begin{array}{l}\text { Entrevistas e documentos legais e } \\
\text { administrativos (legislação, } \\
\text { projetos dos cursos, guias do } \\
\text { aluno, Modelo virtual pedagógica } \\
\text { da UAb) }\end{array}$ & $\begin{array}{l}\text { André (2005); Gil (2008); Coutinho } \\
\text { (2011); UFSC (2013; 2009 a, b, c, } \\
\text { d); Pereira et al. (2007); UFF } \\
\text { (2011) SOUZA, R.A.(2012). } \\
\text { SILVA, A.O et. al. (2012); } \\
\text { entrevistas com docentes e } \\
\text { especialistas em EaD }\end{array}$ \\
\hline Unidades de análise & UFF, UFSC, UFRN, UAb &
\end{tabular}

Fonte: elaborado pelos autores

*UFF - Universidade Federal Fluminense; UFRN -Universidade Federal do Rio Grande do Norte

A partir das considerações supramencionadas, na pesquisa relatada, o processo de investigação partiu da necessidade de conhecer a realidade do contexto em que o fenômeno (educacional) em estudo está inserido. Isso foi possível por meio do estudo de caso coletivo realizados nas seguintes IES: (1) UAb, de Portugal, e (2) UFRN (3) UFF e (4) UFSC, do Brasil. Realizou-se o primeiro estudo na UAb, seguido dos demais. Buscaram-se evidenciar pontos comuns entre esses casos e elucidar pontos particulares de cada um. Em cada caso, considerou-se, a priori, encontrar resultados similares (para constatar características que se sobressaíssem nos Modelos de EaD) e também singulares (para apontar dificuldades e também diferentes alternativas para Modelos de EaD). Objetivou-se gerar proposições teóricas aplicáveis a contextos de cursos a distância de ensino superior. Os dados empíricos se constituíram das experiências vividas por docentes de cursos de Licenciatura a distância dessas IES, os quais conduziram à aproximação da constatação descritiva da realidade, do contexto estudado. 
Para definir a quantidade de unidades de análises (casos), considerou-se o tempo total de duração da pesquisa, a facilidade de contato e a aceitação dos coordenadores dos cursos para a realização da pesquisa, como também a disponibilidade financeira (para a autora da tese permanecer nas IES durante a coleta de dados). Aliado a isso, considerou-se 1 caso com abordagem contrastante (o da UAb de Portugal) e 3 casos com similaridades e diferenças, regidos por um único Sistema brasileiro (UAB). Optou-se por investigar casos com situações: confirmatórias, ou seja, com replicações presumíveis do mesmo fenômeno; teoricamente diferentes e também; contrastantes (YIN, 2005).

Seguindo orientações de Gil (2008), utilizaram-se instrumentos de coleta de dados constituídos de entrevistas orais estruturadas organizadas em um guia composto de questões abertas e de mesma sequencia, nas quais se solicita ao entrevistado respondê-las com suas próprias palavras. Partiu-se do pressuposto de que todos os entrevistados interpretariam as perguntas do mesmo jeito. No Quadro 2, apresenta-se a quantidade de docentes participantes em suas respectivas IES:

Quadro 2-Quantidade de participantes por instituição de ensino
\begin{tabular}{|l|l|l|}
\hline Instituição (Curso) & Total por IES \\
\hline UAb (Educação) & 9 \\
\hline UFF: Matemática & 8 \\
\hline UFRN: Física & 9 \\
\hline \multirow{2}{*}{ UFSC } & Física & 7 \\
\cline { 2 - 3 } & Matemática & 7 \\
\hline TOTAL & & $\mathbf{3 9}$ \\
\hline
\end{tabular}

Em relação à quantidade de entrevistados, consideraram-se as orientações de Moreira (2002), pois, segundo esse autor, é recomendado definir, no máximo, 10 sujeitos de pesquisa. Assim, seguindo essas orientações, considerou-se cada IES como uma unidade de análise específica. Frisa-se que, para validar o guia das entrevistas com os docentes, realizou-se um pré-teste com uma professora da UAb e outro, com uma professora do curso de Licenciatura em Física da UFSC.

A definição dos cursos para investigação das Universidades brasileiras ocorreu a partir da proposição de analisar Modelos de EaD de cursos da área de Ciências Exatas, devido a sua relação com o programa de doutorado no qual a autora da referida pesquisa está vinculada. Quanto à definição dos critérios para a escolha das disciplinas nas universidades brasileiras, considerou-se pertinente selecionar, em cada instituição, duas do departamento de Ciências Exatas, de Matemática ou de Física, e duas do departamento de Educação, as pedagógicas. Com isso, intencionou-se trabalhar com áreas de conhecimento que possuem diferentes aspectos didático-pedagógicos. A partir da definição das disciplinas, buscaram-se seus respectivos docentes para participarem da pesquisa de campo, sendo que ao final, houve predomínio dos participantes das áreas exatas.

Em Portugal - no âmbito de um doutorado sanduíche - realizou-se a coleta de dados em Lisboa, no período de $1^{\mathrm{o}}$ de fevereiro de 2012 a 10 de junho de 2012 . Nesse mesmo período, obtiveram-se duas entrevistas complementares, as quais foram denominadas de "consulta a especialistas em EaD", com a professora Alda Pereira, uma das autoras do Modelo de EaD da UAb, e com o professor Hermano Carmo, professor e um dos fundadores da UAb. Essas entrevistas contribuíram para a compreensão das diretivas da concepção do Modelo de EaD dessa instituição, bem como para fortalecer o aporte teórico na composição e na análise deste estudo.

Nas Universidades brasileiras, a coleta de dados com os docentes ocorreu em diversos momentos, no ano de 2012, conforme Quadro 3: 
Quadro 3 - Período de realização da pesquisa nas universidades brasileiras

\begin{tabular}{|c|c|c|c|}
\hline Instituição & UFF & UFSC & UFRN \\
\hline Período & Setembro/2012 & Julho a novembro/2012 & Agosto/2012 \\
\hline
\end{tabular}

Após a coleta dos dados, seguindo as orientações de Bardin (2011) e de Coutinho (2011), definiram-se as categorias de análise a partir da realização de três fases: a pré-análise, a exploração do material (ambos da pesquisa de campo) e o tratamento dos resultados.

$\mathrm{Na}$ pré-análise, se fez a transcrição das 39 entrevistas obtidas, as quais originaram, aproximadamente, 54 horas de gravação e geraram em torno de 85 páginas de conteúdos transcritos. Em seguida, após uma leitura flutuante ${ }^{1}$ dessas entrevistas, se procedeu à seleção e à organização de trechos das respostas dos participantes, os quais se denominou de asserções, as quais, por sua vez, constituem o estado "original" dos dados empíricos. Além disso, suprimiram-se algumas das perguntas, cujas respostas se consideraram irrelevantes para a pesquisa.

Depois de organizadas, as asserções foram submetidas à revisão de pessoas-chave de cada IES (um docente ou o coordenador de cada licenciatura). Do ponto de vista metodológico, as correções feitas durante esse processo contribuíram com a validade do constructo do estudo.

$\mathrm{Na}$ fase de exploração do material, recortaram-se partes das asserções que mais se repetiram ou que apresentaram particularidades que apontaram aspectos importantes para as proposições de pesquisa e compuseram-se cinco Apêndices ${ }^{2}$ das asserções dos docentes organizadas por curso e respectiva instituição. Neste sentido, Yin (2005) considera que a seletividade das informações contribui com a apresentação de evidências mais significativas e, com isso, é necessário cuidar para não abarrotar o texto com informações secundárias, mas, ao mesmo tempo, possibilitar que a redação contenha dados de sustentação das evidências do autor, para que o leitor crítico possa fazer outras inferências a partir desses dados.

Ainda nessa etapa, por meio de um processo de intensa atenção e compreensão empática, com vistas a deixar de lado ideias próprias da pesquisadora, desenvolveram-se articulações dessas asserções, ou seja, realizou-se um trabalho de compreensão, interpretação e inferência das asserções dos sujeitos entrevistados.

Para melhor compreensão, apresenta-se um exemplo da organização das asserções dos docentes e das articulações dessas asserções. Criou-se um quadro com as questões do guia de entrevista e dispuseram-se suas respectivas evidências das asserções, conforme se exemplifica no Quadro 4.

\footnotetext{
${ }^{1}$ Segundo Bardin (2011), leitura flutuante é o primeiro contato que o pesquisador tem com os dados a serem explorados em uma pesquisa. Nela, surgem as questões norteadoras ou hipóteses baseadas em teorias conhecidas ou que o pesquisador julga pertinente para alcançar suas proposições de investigação.

${ }^{2}$ Os Apêndices foram organizados por curso e IES: (1) física/UFRN, (2) física/UFSC, (3) Matemática/UFSC, (4) Matemática/UFF e (5) Educação/UAb.
} 
Quadro 4 - Exemplo de asserção dos docentes

\begin{tabular}{|c|c|}
\hline \multicolumn{2}{|c|}{ Questão: Como você definiria o Modelo de EaD da UFRN } \\
\hline Professor(a) & Asserção do docente \\
\hline d1_UFRN_FIS & $\begin{array}{c}\text { Um Modelo semipresencial, os alunos têm um apoio físico nos polos. [...] nós não } \\
\text { temos entrada regular. [...] Em algumas disciplinas, já fica mais complicado. Por } \\
\text { exemplo, no primeiro semestre, há disciplinas comuns a outros cursos, com 600 } \\
\text { alunos. São 3 professores e 3 tutores a distância. }\end{array}$ \\
\hline d2_UFRN_FIS & $\begin{array}{c}\text { Da maneira como ele foi estruturado e está apto a funcionar, é individualizado, é } \\
\text { aula particular. Se o aluno tiver interesse, ele tem aula particular durante o } \\
\text { semestre inteiro. }\end{array}$ \\
\hline d4_UFRN_FIS & $\begin{array}{c}\text { No caso da disciplina de Estágio, não temos muitos alunos; no máximo, são 70 } \\
\text { alunos. Mesmo assim, temos uma interação mais individualizada do que coletiva } \\
\text { (de massa). Mas, se tivesse mais alunos, eu não vislumbro como poderia fazer } \\
\text { uma orientação de mesma forma. No estágio, não tem como eu ir pro Modelo } \\
\text { industrial utilizando o instrumento que uso atualmente, que é o diário reflexivo. }\end{array}$ \\
\hline
\end{tabular}

Conforme Quadro 4, para identificar cada docente entrevistado, se definiram códigos representativos: utilizou-se a letra d (inicial de docente), seguida do número sequencial do(a) docente entrevistado(a) e da sigla da sua respectiva instituição e curso. Nesse sentido, d4_ UFRN_FIS, por exemplo, referencia o $4^{\circ}$ docente entrevistado do curso de Licenciatura em Física da UFRN. Apresenta-se, a seguir, um exemplo de uma das asserções de três docentes, mencionados no quadro, cuja articulação constituiu o seguinte corpus, relacionado à maneira como os professores definem o Modelo de EaD de seu curso:

Os docentes possuem formas distintas para definir o Modelo de EaD do curso a distância de Licenciatura em Física da UFRN. Entre elas, destacam-se: um Modelo semipresencial; um Modelo que tem condições de estabelecer interações pedagógicas, devido ao número de aluno não ser elevado, por não haver uma entrada regular de alunos. Porém, em disciplinas cuja quantidade de alunos se aproxima de 600, atendidos por 3 professores e 3 tutores a distância, o Modelo se aproxima do industrial, de massa; um Modelo com ensino "individualizado", com características de aula particular. Essa característica pode ser evidenciada, por exemplo, na disciplina de Estágio, que está estruturada de forma que seria dificil tratá-la nos moldes de ensino de massa, pois o atendimento e a interação, na maioria das vezes, têm que ser individualizados, considerando as particularidades das dúvidas, das situações e das dificuldades inerentes a cada projeto de intervenção. $O$ instrumento que atualmente é utilizado, o diário reflexivo, conduz claramente a um Modelo que prima pela interação e pelo cuidado particular de acordo com o contexto de cada aluno.

Finalmente, a terceira etapa, tratamento dos resultados, compõe-se de articulações das asserções dos docentes em 3 etapas: na primeira, elaborou-se um corpus dessas articulações por caso (curso/instituição) que, em seguida, passaram por análises e interpretações preliminares e organização. $\mathrm{O}$ resultado desse processo contribuiu com a definição das categorias de análise apresentadas a seguir, juntamente com suas respectivas proposições e questões de investigação:

- Mudanças paradigmáticas, definição, satisfação e valorização do Modelo de EaD na concepção dos docentes: investigam-se: quais mudanças de paradigmas são destacadas pelos docentes na sua atuação entre a Educação presencial e na EaD? Como os docentes definem o Modelo de EaD de seu curso? Qual o grau de satisfação dos docentes com relação ao Modelo de EaD de seu curso? Quais aspectos do Modelo de EaD, são mais valorizados pelos docentes?

- Recursos didáticos: identificam-se quais recursos didáticos são utilizados nos cursos bem como se inferiu qual deles predomina. Questiona-se: qual é o meio de acesso 
aos conteúdos do curso mais utilizados pelos alunos: o impresso ou o digital? Quais são os principais recursos tecnológicos utilizados pelos docentes para desenvolver os processos de ensino e de aprendizagem?

- Interação pedagógica: analisa-se como esta componente se estabelece nas práticas docentes. Questiona-se: a qualidade da Educação está relacionada ao grau de interação entre aluno e professor? Como a interação se estabelece nos Modelos de EaD de IES brasileiras?

- Aprendizagem colaborativa: identificam-se quais estratégias de aprendizagem colaborativa são realizadas nas licenciaturas. Questiona-se: Quais estratégias de aprendizagem colaborativa são realizadas nas Licenciaturas? Quais recursos são utilizados?

Em seguida, na segunda etapa, a partir dos resultados obtidos, contextualizou-se um único corpus textual para cada categoria de análise constituído das similaridades, pontos convergentes e divergentes das articulações das asserções dos docentes das três IES brasileiras e outro dos docentes da UAb, sendo que este texto se estabeleceu como a fonte de dados para a análise (terceira etapa), considerando as cinco categorias de análise, supracitadas e respectivas proposições de pesquisa.

Ao final, com o resultado da análise do estudo de caso coletivo, estima-se contribuir com a caracterização de Modelos de $\mathrm{EaD}$ no ensino superior brasileiro na perspectiva de docentes.

\section{Considerações finais}

Ao optar por uma Metodologia de pesquisa com predomínio da natureza qualitativa, cuja principal fonte de dados empíricos se constituiu de entrevistas, constataram-se nessa experiência, limitações e também potencialidades. Se por um lado, a quantidade de entrevistados dificultou a organização dos dados, por outro, contribuiu de forma significativa com a convergência de ideias a partir da compreensão, pontos de vista e experiências de cada docente em suas respectivas disciplinas.

Um dos desafios encontrados, diante das 85 páginas de transcrição, foi apresentá-las sem correr riscos de poluir o texto com quadros compostos pelas asserções dos docentes e também não tornar a leitura do texto cansativa e as articulações das asserções repetitivas. Ao mesmo tempo, se teve a preocupação de dar veracidade aos dados empíricos, razão pela qual se optou por apresentar, no texto principal da tese, apenas as articulações das asserções finais. Suprimiu-se a apresentação dos quadros com os recortes que originaram as articulações, mas se fez a apresentação dos mesmos em Apêndices, por IES/Curso para consultas aprofundadas.

Constatou-se, nas asserções dos docentes, muitas aproximações, havendo casos em que foram suprimidas devido à irrelevância à pesquisa. Salienta-se que, mesmo sendo a pesquisa de caráter principalmente qualitativo, foi possível mensurar dados e, com isso, geraram-se oportunidades de análise quantitativas, como, por exemplo, identificar quais recursos são mais utilizados nas licenciaturas e qual o tempo de atuação na docência presencial e EaD, e suas relações com mudanças paradigmáticas.

Nas entrevistas, destacaram-se questões que conduziram os entrevistados a apresentar suas interpretações e definições sobre os Modelos de $\mathrm{EaD}$ que utilizam, sendo que, algumas conduziram a interpretações para novas pesquisas, além de que forneceram novas evidências. Não obstante, Yin (2005) orienta para o cuidado de o pesquisador não se tornar refém dos entrevistados e buscar comprovar essas novas evidências em outras fontes que as confirmem ou as corroborem. 
Optou-se por realizar o processo de organização dos dados empíricos utilizando um editor de texto sem apoio de softwares para esta finalidade. Entretanto, alternativas de software para organização de dados qualitativos como, por exemplo, o NVivo, e WebQDA ${ }^{3}$, podem ser úteis para este tipo de metodologia de pesquisa que geram grande quantidade de textos para análise e interpretação.

Como ponto frágil, constatou-se que seria de grande valia ter realizado um estudo de caso piloto em um dos cursos da UFSC, devido à proximidade da autora da presente pesquisa e na facilidade de retornar a essa instituição na iminência de validar os sujeitos, instrumentos e técnicas de coleta dos dados empíricos. Yin (2005) enfatiza a importância da realização de um estudo de caso piloto com características relevantes à pesquisa (um caso muitíssimo complicado), para, em seguida, realizar os outros. Esse procedimento conduz ao aprimoramento, validação ou revisão do projeto de pesquisa inicial, bem como à eficiência da pesquisa.

Salienta-se que esta pesquisa encontra-se na sua fase final, na análise dos dados e apresentação dos resultados. Julga-se que o método, os processos e as técnicas adotadas, apesar das limitações supramencionadas, poderão conduzir ao alcance das proposições da pesquisa.

\section{Referências}

André, M. E. D. A (2005). Estudo de Caso em Pesquisa e avaliação educacional. Brasília: Liber Livro Editora.

Bardin, L. (2011). Análise de conteúdo. Edição revista e ampliada. Trad. Luís Antero Reto, Augusto pinheiro. São Paulo: Edições 70.

Coutinho, C. (2011) Metodologias de Investigação em Ciências Humanas. Coimbra: Almedina.

Denzin, N.; Lincoln, Y. (2006). O planejamento da pesquisa qualitativa. Porto Alegre: Bookman.

Garrison, G. R. (2000) International Review of Research in Open and Distance Learning, ISSN: 1492-3831, Vol. 1, No. 1.

Garrison, R. D., \& Anderson, T. (2003). E-learning in the 21st century: A framework for research and practice. New York: Routledge Falmer.

Gil, A. C. (2008). Como elaborar projetos de pesquisa. 5a . ed. São Paulo: Atlas.

Gomes, M. J. (2008) Na senda da inovação tecnológica na Educação a Distância. Revista portuguesa de pedagogia. Ano 42-2, p. 181-202.

Moore, M., Kearsley, G. (2007) Educação à distância: uma visão integrada.Tradução Roberto Galman. São Paulo: Thomson Learning.

Moreira, D. A.(2002) O Método Fenomenológico na Pesquisa. São Paulo: Thompson Pioneira.

Pereira, A., Quintas-Mendes, A.,Mota, J., Morgado,L., Aires,L. (2007). Modelo pedagógico virtual da Universidade Aberta : para uma universidade do futuro. Lisboa: Universidade

\footnotetext{
${ }^{3}$ Informações disponíveis em https://www.webqda.com/
} 44 
Aberta, p. 1-112. Disponível em https://repositorioaberto.uab.pt/handle/10400.2/1295. Acesso em 23 de jan. de 2013.

Silva, A.O., Brito, A.C.F., Fernandes, J.D.G., Lucena, G.B.O. (2012). Uma experiência: criação e funcionamento do curso de química na modalidade Educação a Distância da UFRN. In:A pratica da Educação a Distância na Universidade Federal do Rio Grande do Norte. Neto, J.C.T e Paiva, M.C.L. (orgs). Natal: EDUFRN. 294 P. $1^{\text {a }}$. edição. Pgs. 23-44.

Souza, R.A.(2012). Tutor presencial em EaD: qual é o papel desse indivíduo?. In:A pratica da Educação a Distância na Universidade Federal do Rio Grande do Norte. Neto, J.C.T e Paiva, M.C.L. (orgs). Natal: EDUFRN. 294 P. 1ª edição. Pgs. 69-88.

Stake, R.E. (2005). Cases Studies. In. Denzin, N.K.; Lincoln, Y.S. (ed.) Handbook of qualitative research. London: Sage, p. 435-454.

UFSC. 2009a. Guia do aluno. Disponível em https://ead.ufsc.br/matematica/files/2009/10/MTMGuia-do-Aluno.pdf. Acessado em 28.08.2012.

UFSC. 2009b. Guia do tutor. Disponível em https://ead.ufsc.br/matematica/files/2009/08/MTM_GuiaTutor_FINAL-1.pdf

UFSC. 2009c. UAB no Brasil e na UFSC. Disponível em https://ead.ufsc.br/matematica/uabno-brasil-e-na-ufsc/ . Acessado em 28.08.2013.

UFSC. 2009d. Projeto Político Pedagógico do curso de Licenciatura em Matemática na Modalidade a distância. Universidade Federal de Santa Catarina, Florianópolis, SC Brasil.

UFSC. 2013. Projeto Político Pedagógico do curso de Licenciatura em Física na Modalidade a distância. Disponível em https://ead.ufsc.br/fisica/projeto-politico-pedagogico-20013/. Acessado em 28.08.2013.

UFF. (2011). Projeto Pedagógico do Curso de Licenciatura em Matemática a Distância, Instituto de Matemática e Estatística, Universidade Federal Fluminense, Niterói, RJ Brasil.

Yin, R.K. (2005). Estudo de caso :planejamento e métodos /Robert K. Yin; tradução: Daniel Grassi. - $3^{\mathrm{a}}$ ed. - Porto Alegre: ArTmed, - 205 p. 